\title{
Eternal inflation, bubble collisions, and the persistence of memory
}

\author{
Jaume Garriga \\ Departament de Fisica Fonamental, Universitat de Barcelona, Marti i Franques 1, 08028 Barcelona, Spain
}

Alan H. Guth

Center for Theoretical Physics, Laboratory for Nuclear Science and Department of Physics, Massachusetts Institute of Technology, Cambridge, Massachusetts 02139, USA

\begin{abstract}
Alexander Vilenkin
Institute of Cosmology, Department of Physics and Astronomy Tufts University, Medford, Massachusetts 02155, USA
\end{abstract}

(Received 2 July 2007; published 27 December 2007)

\begin{abstract}
A "bubble universe" nucleating in an eternally inflating false vacuum will experience, in the course of its expansion, collisions with an infinite number of other bubbles. In an idealized model, we calculate the rate of collisions around an observer inside a given reference bubble. We show that the collision rate violates both the homogeneity and the isotropy of the bubble universe. Each bubble has a center which can be related to "the beginning of inflation" in the parent false vacuum, and any observer not at the center will see an anisotropic bubble collision rate that peaks in the outward direction. Surprisingly, this memory of the onset of inflation persists no matter how much time elapses before the nucleation of the reference bubble.
\end{abstract}

DOI: 10.1103/PhysRevD.76.123512

PACS numbers: 98.80.Cq, 98.80.Qc

\section{INTRODUCTION}

In a theory with different metastable vacua, such as the landscape of string theory [1,2], the process of eternal inflation may lead to a "multiverse" where different regions of space-time are occupied by different vacua. A region occupied by vacuum $\mathrm{A}$ may spawn regions of other vacua $B, C, D \ldots$ which are adjacent in field space. Daughter regions will in turn spawn their offspring, and so on, producing an infinite tree of "pocket universes."

Vacua can evolve by a mix of semiclassical tunneling and stochastic evolution, but in this paper we will consider only tunneling. In that case, bubbles form by random nucleation and then start to expand into the parent vacuum with constant acceleration. The interior of the growing bubble has the geometry of an open FriedmannRobertson-Walker (FRW) universe [3], and (assuming that a short period of slow-roll inflation flattens it out to satisfaction) we may entertain the possibility that we live in one of such "bubble universes" [4].

The above description, however, is incomplete because it ignores collisions with other bubbles. Collisions may be quite rare if the nucleation rates are small. Nonetheless, a bubble expands for an infinite amount of time, and will collide and merge with an infinite number of other bubbles, forming an ever growing "cluster."

We should therefore reevaluate the naive picture of a smooth FRW universe on large scales. In particular, we may ask what fraction of the FRW universe remains unaffected by collisions. As we shall see, only a set of measure zero remains unaffected. If this is supposed to describe our local universe, there seems to be reason for concern. Why have not we seen any collisions yet? Do we have much time left until we are blown away by a collision? What is the expected distance to the nearest point in our FRW time slice which has already been hit by another bubble? The purpose of the present paper is to explore some of these issues.

In the course of this investigation we have stumbled upon a rather remarkable result. We find that the rate of collisions around a typical observer in the bubble universe is anisotropic, and the origin of this anisotropy is related to the beginning of false vacuum inflation.

A metastable inflationary de Sitter phase can be eternal to the future, but not to the past. More precisely, the inflating region of space-time is geodesically pastincomplete [5], and therefore some initial conditions must be specified on the past boundary of this region. For instance, we may posit that at some initial time, a given large region of space is in false vacuum. The congruence of geodesics normal to the initial time surface defines a preferred frame, with respect to which velocities can be defined.

The standard lore is that this preferred frame is not important, and that the initial conditions are soon forgotten. This may be true for geodesic observers in the false vacuum phase, whose velocity with respect to the preferred congruence redshifts exponentially with time. For those "comoving" observers, memory of the initial surface is lost as we push the surface far away into the remote past. However, when a bubble of a new phase forms in the original false vacuum, the congruence of observers in the FRW open universe includes observers with velocities arbitrarily close to the speed of light relative to the preferred congruence. Such individuals are not "far" from 
some points on the initial surface, and may have a chance to detect some information about the beginning of inflation.

The plan of the paper is the following. In Sec. II we describe the geometry and statistics of collisions onto a given reference bubble. In Sec. III, we consider the anisotropies in the bubble distribution as seen by observers inside a reference bubble. The origin of these anisotropies is further discussed in Sec. IV in a simplified context, where the observer is in false vacuum rather than inside the bubble. Section V is devoted to conclusions. Some technical details are left for the appendices.

\section{COLLISIONS ONTO A REFERENCE BUBBLE}

Here, we shall derive some basic results concerning the distribution of collisions impinging on a given reference bubble. We begin with a description of the basic setup we shall consider.

It is convenient to use flat de Sitter coordinates to describe the background inflating false vacuum,

$$
d s^{2}=d t^{2}-e^{2 H t}\left(d r^{2}+r^{2} d \Omega^{2}\right) .
$$

To simplify the equations, we shall choose units so that

$$
H=1 \text {. }
$$

To simplify matters further, we shall assume that the vacuum energy density inside the bubbles is nearly the same as that outside (at least for a sufficiently long time after bubble nucleation) and that the gravitational effect of bubble walls is negligible. Then the metric is

$$
d s^{2}=d t^{2}-e^{2 t}\left(d r^{2}+r^{2} d \Omega^{2}\right)
$$

in the entire space-time region of interest.

It is also useful to consider the "embedding" of de Sitter space as a timelike hyperboloid of unit radius, in a 5 dimensional Minkowski space, whose rectangular coordinates are labeled $V, W$ and $\vec{X}=(X, Y, Z)$ :

$$
\vec{X}^{2}+W^{2}-V^{2}=1 \text {. }
$$

These are related to the flat chart coordinates $t$ and $\vec{x}=$ $(x, y, z),(r \equiv|\vec{x}|)$, through

$$
W-V=e^{-t}-e^{t} r^{2}, \quad W+V=e^{t}, \quad \vec{X}=e^{t} \vec{x} .
$$

Let us consider a "reference" bubble that nucleates at $t=$ $r=0$ (we shall sometimes call this "our" bubble). In the embedding coordinates, this corresponds to

$$
W=1, \quad V=\vec{X}=0 .
$$

The bubble geometry is symmetric under boosts which have the nucleation event as a fixed point. These form an $O(3,1)$ group of isometries.

The interior of the bubble (or more precisely, the interior of the light cone from the nucleation event) is described by the line element

$$
d s^{2}=d \tau^{2}-\sinh ^{2} \tau\left(d \xi^{2}+\sinh ^{2} \xi d \Omega^{2}\right) .
$$

The coordinates $(t, r)$ and $(\tau, \xi)$ are related by

$$
\begin{gathered}
e^{t}=\cosh \tau+\cosh \xi \sinh \tau, \\
e^{t} r=\sinh \xi \sinh \tau .
\end{gathered}
$$

We set the initial condition that there are no bubbles at some $t=t_{i}$. This breaks the residual $O(3,1)$ invariance of the bubble, which is responsible for the homogeneity and isotropy of our FRW universe. Consequently, not all observers who live in the open FRW universe will see the same.

\section{A. Collisions around the observer at $\boldsymbol{\xi}=\mathbf{0}$}

Let us now concentrate on the distribution of collisions around the point $\xi=\xi_{\mathrm{obs}}=0$, which is at the origin of the open FRW hyperboloid. We leave the consideration of typical observers, who live far away from the origin, to Secs. II B and III.

The distribution around $\xi_{\text {obs }}=0$ will of course be isotropic. In particular, we shall be interested in the typical distance at which we might expect the nearest collision. In what follows, we shall calculate the probability $P(\xi, \tau)$ that no collisions with other bubbles have affected a spherical region of radius $\xi$ around the origin, on a hypersurface of constant $\tau$.

As a warm up exercise, let us consider the following question. Assuming that the point $t=r=0$ is still in false vacuum, what is the probability that some bubble will have hit the surface $t=0$ at some $r \leq r_{0}$ ? The relevant quantity is the 4 volume inside the past light cone of the circle $r<$ $r_{0}$ minus the 4 volume inside the past light cone of the origin $r=0$ (see Fig. 1). The physical radius of the past light cone from the origin is $R_{0}(t)=1-e^{t} \quad(t<0)$, whereas the radius of the past light cone from the circle of radius $r_{0}$ is $R_{1}(t)=R_{0}(t)+r_{0} e^{t}$. The volume of the gray shaded region in Fig. 1 is thus given by

$$
\begin{aligned}
\mathcal{V}_{4}\left(r_{0}, t_{i}\right) & =\frac{4 \pi}{3} \int_{t_{i}}^{0}\left(R_{1}^{3}-R_{0}^{3}\right) d t \\
& =\frac{2 \pi}{9}\left(6 r_{0}+3 r_{0}^{2}+2 r_{0}^{3}\right)+O\left(e^{t_{i}} r_{0}\right) .
\end{aligned}
$$

Note that this is finite (for finite $r_{0}$ ) even in the limit when the initial surface is pushed all the way to $t_{i} \rightarrow-\infty$. For a region of a Hubble size, we have

$$
\mathcal{V}_{4}\left(1, t_{i} \rightarrow-\infty\right)=\frac{22 \pi}{9} \text {. }
$$

Hence, the probability of having a bubble one Hubble distance away from ours at $t=0$ is of order of the nucleation rate per unit volume $\lambda$, which we shall assume to be small $(\lambda \ll 1$ in the units where $H=1)$. The distance to the nearest bubble at $t=0$ can be estimated from the 


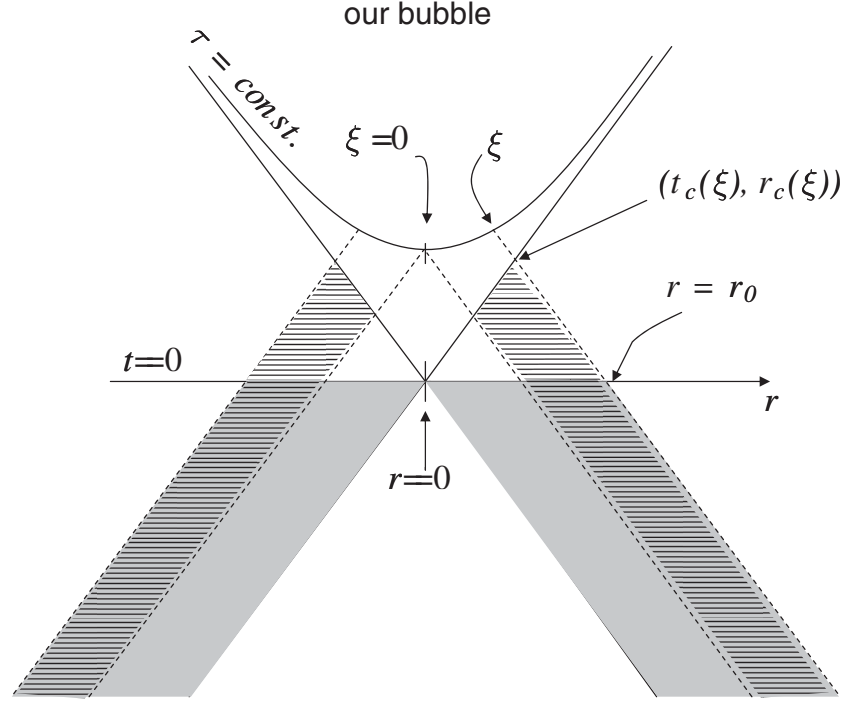

FIG. 1. Region in gray shade, $V_{4}\left(r_{0}\right)$ can nucleate bubbles which will be within the distance $r_{0}$ from the origin (on the flat surface $t=0$ ). Here, we assume that the point $r=t=0$ is still in false vacuum. Region in horizontal stripes, $V_{4}(\xi, \tau)$, can nucleate bubbles which will collide with ours, and which will be visible within a coordinate radius $\xi$ from the origin (on the $\tau=$ const hypersurface). Here, we assume that the center point $\xi=0$ has not yet been hit by any bubble.

condition $\lambda \mathcal{V}_{4} \sim 1$. From Eq. (10), this distance will be of the order $r_{0} \sim \lambda^{-1 / 3}$.

Next, we may consider the same question on a $\tau=$ const hyperboloid. Past directed radial null rays define a mapping between points on the hyperboloid at some distance $\xi$ and points on the "bubble cone," by which we mean the future light cone from the nucleation event. The bubble cone is given by the equation

$$
r(t)=1-e^{-t}, \quad(t>0) .
$$

The flat chart coordinates of points on the hyperboloid can be found from Eq. (9). The past directed outward radial null geodesic from a point $(\xi, \tau)$ is given by

$$
r(t)=e^{-t}+F(\tau, \xi),
$$

where

$$
F=\frac{\sinh \xi \sinh \tau-1}{\cosh \tau+\cosh \xi \sinh \tau} .
$$

Note, in particular, that for $\xi=0$, we have $F=-e^{-\tau}$, whereas for large $\xi$ and $\tau$, we have

$$
F(\xi, \tau) \approx 1-2 e^{-\xi}+2 e^{-2 \xi}-4 e^{-\tau-\xi} \quad(\xi, \tau \gg 1) .
$$

The intersection of the null geodesic (12) with the bubble cone (11) is at

$$
e^{-t_{c}}=\frac{1-F}{2}, \quad r_{c}=\frac{1+F}{2} .
$$

(Incidentally, the intersection with the plane at $t=0$ is at $r_{0}=1+F<2$.)

It will be very convenient to use Eq. (12) as the definition for a change of variables, to replace $r$ in favor of $F$. The different values of $F$ can be thought of as labeling the different past directed outward radial null rays emanating from a given $\xi$, on a $\tau=$ const hyperboloid, as indicated by Eq. (13). More explicitly, we introduce

$$
a \equiv e^{t}, \quad F \equiv r-e^{-t},
$$

in terms of which the metric reads

$$
d s^{2}=2 d a d F-a^{2} d F^{2}-(1+a F)^{2} d \Omega^{2} .
$$

This form of the metric is completely regular at $a=0$ (which corresponds to the boundary of the flat coordinatization). In fact, the new chart (17) covers the whole of de Sitter space, when we let the coordinates vary in the range $-\infty<a<\infty,-\infty<F<\infty$, with the restriction $a F>-1$. Negative values of $a$ correspond to the part of de Sitter space not covered in a flat chart.

Let us assume that, on a given $\tau=$ const hyperboloid, the origin $\xi=0$ has not yet been hit by a bubble, and let us ask what is the typical distance at which we may expect the nearest collision. The probability that no collisions have affected a region of coordinate radius $\xi$ around the origin is given by

$$
P\left(\xi, \tau, t_{i}\right)=e^{-\lambda V_{4}},
$$

where $\lambda$ is the nucleation rate, and $V_{4}$ is the relevant 4 volume to the past of a spherical region of radius $\xi$ on the hyperboloid. Here, we must subtract the contribution from the interior of the past light cone of $\xi=0$ (this is because we assume $\xi=0$ has not been hit by any bubbles), and also the intersection with the interior of our bubble, since we assume that no new bubbles can nucleate inside of our bubble. We may express this 4 volume as

$$
\begin{aligned}
V_{4}\left(\xi, \tau, t_{i}\right) & =4 \pi \int e^{3 t} r^{2} d r d t \\
& =4 \pi \int e^{3 t}\left(e^{-t}+F\right)^{2} d F d t \\
& =4 \pi \int(1+a F)^{2} d a d F
\end{aligned}
$$

The evaluation of $V_{4}$ is simplest in the $(F, a)$ coordinates. This is because the light cones are bound by null geodesics with $F=$ const. Hence, the integration limits for $F$ in (19) are independent of $a$,

$$
-e^{-\tau}<F<F(\tau, \xi) .
$$

The lower limit corresponds to the center of the hyperboloid $\xi=0$. The integration range for $t$ depends on $F: t_{i}<$ $t<t_{c}(F)$, where $t_{c}$ is given in (15). Here we are approximating the boundary of our bubble by the "bubble cone," which is justified when the size of bubbles at the time of nucleation is much less than the Hubble radius. In terms of 
$a=e^{t}$, the range is given by

$$
a_{i}<a<2 /(1-F) .
$$

Performing the double integral, we have

$$
V_{4}\left(\xi, \tau, t_{i}\right)=\frac{8 \pi}{3}\left[\frac{2 F}{(1-F)^{2}}-\ln (1-F)+O\left(a_{i} F\right)\right]_{F=-e^{-\tau}}^{F(\xi, \tau)},
$$

where the upper limit is given by (13). Note that the 4 volume takes a finite value in the limit when the initial surface is pushed into the remote past $a_{i} \rightarrow 0$.

Defining $V_{4}(\xi, \tau) \equiv \lim _{t_{i} \rightarrow-\infty} V_{4}\left(\xi, \tau, t_{i}\right)$, we find

$$
\begin{aligned}
V_{4}(\xi, \tau)= & \frac{8 \pi}{3}\left\{e^{\xi} \sinh \xi \tanh ^{2}\left(\frac{\tau}{2}\right)\right. \\
& \left.+\ln \left[1+\frac{1}{2}\left(1-e^{-\tau}\right)\left(e^{\xi}-1\right)\right]\right\} .
\end{aligned}
$$

In particular, for $\tau \gg 1$, we have

$$
\begin{aligned}
V_{4}(\xi, \tau) \approx 4 \pi \xi+O\left(\xi^{2}\right) & (\xi \ll 1), \\
V_{4}(\xi, \tau) \approx \frac{4 \pi}{3} e^{2 \xi}+O(\xi) & (\xi \gg 1) .
\end{aligned}
$$

The typical coordinate distance to the nearest bubble is found from $\lambda V_{4} \sim 1$, and for small nucleation rates, $\lambda \ll$ 1 , it is of order

$$
\Delta \xi \sim \ln (1 / \lambda) .
$$

Note that this is independent of $\tau$. This is to be expected, since (for $\tau \gtrsim 1$ ) the products of collision are "frozen in" with the expansion of the universe during the period of inflation inside the bubble. Here, for simplicity, we have approximated this period as a de Sitter phase with the same expansion rate as the false vacuum. In a more realistic case we should consider a period of slow-roll inflation, followed by a standard decelerating phase. If this phase leads to a Minkowski vacuum, all products of collision would gradually fall within the horizon, and eventually hit any observer inside the bubble. (This is explicitly shown at the end of Appendix A, where we calculate the rate at which an observer in Minkowski vacuum would see new bubbles falling into his field of view.) On the other hand, our own universe does not seem to be approaching a Minkowski vacuum. Rather, it appears to be accelerating again, and collisions with other bubbles may forever remain hidden behind the cosmological horizon.

The curvature radius of the FRW universe corresponds to $\Delta \xi \sim 1$. Current observational bounds on the spatial curvature imply [6] $\left(a_{0} H_{0}\right)^{-2}=|1-\Omega| \lesssim 10^{-2}$, where the subindex 0 indicates the present time. The coordinate size of the observable universe is given by $\Delta \xi_{0} \sim$ $\left(a_{0} H_{0}\right)^{-1}$. Hence, the observer at the center of the hyperboloid $\xi_{\text {obs }}=0$ can only see out to coordinate distances $\Delta \xi_{0} \lesssim 10^{-1}$. Using (18) and (22), the probability that this observer may see any collisions at all is given approximately by

$$
1-P\left(\Delta \xi_{0}\right) \approx 1-e^{-4 \pi \lambda \Delta \xi_{0}} \lesssim \lambda \ll 1 .
$$

This issue will be discussed further in Sec. III, and also in Appendix A. In the appendix we show that the asymptotic expressions (22) and (23) are valid also for the relevant four volume in the neighborhood of any observer (at $\xi_{\text {obs }} \gg 1$ ) who has not yet been hit by a bubble. This means that we are very unlikely to be hit by a bubble at any time in the future.

The expected physical distance to the collision nearest to $\xi=0$, at the time when inflation ends, can be estimated as

$$
d=Z \ln (1 / \lambda) \text {, }
$$

where $Z \sim \sinh \tau_{f}$ is the slow-roll expansion factor $\left(\tau_{f}\right.$ is the time at the end of inflation inside the bubble). Here we have worked in a simplified scenario where $H$ is the same in false vacuum as it is in the period of slow-roll inflation inside the bubble. In the general case, the expression for $d$ will be more complicated, but the dependence on $Z$ and $\lambda$ will be similar.

\section{B. Fractal dimension of the bubble universe}

The worldline of a given point $(\theta, \phi)$ on the wall of the reference bubble will sooner or later be hit by other bubbles, with probability equal to one. This follows from the fact that there is a finite probability per unit time $t$ for this worldline to be hit by other bubbles (see Sec. IV for a rigorous derivation of this statement). Hence, the fraction of the bubble wall area that makes it to future infinity without collisions is a set of measure zero.

The effect of collisions onto the reference bubble propagates into the open FRW universe. On the hyperbolic slice $\tau=$ const, each collision affects a "wedge" shaped region, whose tip points towards the origin and which extends all the way to $\xi \rightarrow \infty$, spanning a finite asymptotic solid angle at spatial infinity. Let us denote by $\xi_{c}$ the distance from the tip of the wedge to the origin, and let us calculate the asymptotic angular radius of the wedge, $\theta_{w}$, as a function of $\xi_{c}$.

It is convenient, again, to use the coordinates $a, F$ of the chart (17). A bubble that nucleates at time $a$ has asymptotic comoving radius $1 / a$ at future infinity. A bubble nucleating at point $F$ will have its center displaced a distance $r=$ $F+a^{-1}$ from the origin, and its boundary at future infinity will have comoving Cartesian coordinates satisfying:

$$
x^{2}+y^{2}+\left(z-F-a^{-1}\right)^{2}=a^{-2} .
$$

(Here we have assumed that the center is on the $z$ axis.) The future boundary of the reference bubble (which nucleates at $t=r=0$ ) is given by

$$
x^{2}+y^{2}+z^{2}=1 .
$$

The intersection of both is at 


$$
z=\cos \theta_{w}=\frac{1}{2} \frac{a+2 F+a F^{2}}{1+a F} .
$$

Note that $\theta_{w}$ corresponds to the asymptotic angular radius of wedges corresponding to bubbles nucleated at $(a, F)$.

On a hyperboloid with $\tau \gtrsim 3$, large $\xi$ corresponds to $1-F \approx 2 e^{-\xi}(1+\cosh \tau) / \sinh \tau \approx 2 e^{-\xi}$. From (19), the bubbles which affect this region nucleate in a four-volume dominated by the largest possible $a$,

$$
a \sim \frac{2}{1-F} \sim e^{\xi} .
$$

From (28), The typical angular size of wedges whose tip is at $\xi_{c} \gg 1$ is therefore

$$
\theta_{w}\left(\xi_{c}\right) \sim e^{-\xi_{c}} \ll 1 .
$$

Let us denote by $\Omega_{u}(\xi)$ the solid angle which remains unaffected by collisions out to a distance $\xi$ from the origin. As $\xi$ is increased, more and more wedges accumulate, each one removing a solid angle $2 \pi\left[1-\cos \theta_{w}\right]$ from $\Omega_{u}$. The loss of solid angle due to wedges whose tip is in the interval $d \xi$ is given by

$$
d \Omega_{u}(\xi) \approx-2 \pi\left[1-\cos \theta_{w}\right] \lambda d V_{4} \sim-\lambda e^{-2 \xi} d V_{4},
$$

where $d V_{4}$ is the region of four volume to the past of the interval $d \xi$, in the unaffected portion of the sphere. Here, we are making the approximation that wedges do not overlap with each other. Also, we shall assume that a wedge whose tip is at $\xi_{c}$ depletes a solid angle equal to the corresponding asymptotic value (at $\xi \rightarrow \infty$ ) for all $\xi>$ $\xi_{c}$. The four volume is approximately given by Eq. (19), where $4 \pi$ is replaced by $\Omega_{u}(\xi)$. Using (28), we have

$$
\begin{aligned}
2 \pi\left[1-\cos \theta_{w}\right] d V_{4}= & \pi \Omega_{u}(\xi)\left[\int_{a=0}^{2 /(1-F)}(1-F)\right. \\
& \times[2-a(1-F)](1+a F) d a] d F \\
= & \frac{2 \pi(3-F)}{3(1-F)} \Omega_{u}(\xi) d F \\
\approx & \frac{4 \pi}{3} \Omega_{u}(\xi) d \xi
\end{aligned}
$$

where in the last step we have used $F \approx 1-2 e^{-\xi}$.

Combining Eqs. (30) and (31), the solid angle which remains unaffected by collisions as a function of $\xi$ is given by

$$
\Omega_{u}(\xi) \approx 4 \pi e^{-\kappa \xi}, \quad(\xi \gg 1),
$$

where $\kappa \approx(4 \pi / 3) \lambda$. The volume element on a spatial section of an open FRW is given by $d V^{\mathrm{FRW}}=$ $4 \pi \sinh ^{2} \xi d \xi$. The volume which is unaffected by bubbles

$$
d V=\Omega_{u}(\xi) \sinh ^{2} \xi d \xi \sim 2 \pi e^{(2-\kappa) \xi} d \xi,
$$

is therefore unbounded and dominated by large distances from the origin (assuming, of course, that $\kappa \ll 1$ ).
Nevertheless, the unaffected volume fraction tends to zero in the limit $\xi \rightarrow \infty$. The unaffected volume fraction will be recalculated in the next section, using a method which is exact in the context of our idealized model.

The fact that the unaffected part of the volume is a set of measure zero might seem to be reason for concern. Nevertheless, if we pick a random point in the unaffected region, then the closest hit is likely to be quite far away. Indeed, the number of bubbles hitting in the interval $d \xi$ is given by $d N=\lambda d V_{4}$. Using (29), (31), and (33) we find that for large $\xi$,

$$
d N \sim \lambda d V .
$$

Let us now pick a random point in the unaffected region, at $\xi \gg 1$, and let us choose it as the origin of open FRW coordinates. A spherical region of radius $\Delta \xi \gg 1$ around that point has coordinate volume $V \sim e^{2 \Delta \xi}$. Using (34), the expected distance to the nearest bubble will be of order

$$
\Delta \xi \sim \ln (1 / \lambda) .
$$

This is the same result we found in the previous Sec. II A, for the distribution around the privileged point at the center of the hyperboloid. The coordinate distance (35) is large enough that we should not be too concerned about the hazard of future collisions.

Finally, let us characterize the fractal dimension of the unaffected part of the bubble universe. Looking outward from the center of the hyperboloid, we see finer and finer "wedges" carving away the solid angle as we increase $\xi$. If we define a smearing angle $\epsilon \equiv e^{-\xi}$, and ignore structures smaller than $\epsilon$, the unaffected solid angle is $\Omega_{u}(\xi) \approx$ $4 \pi \epsilon^{\kappa}$. The number of sets of angular radius $\epsilon$ which is needed to cover this region is $n \sim \epsilon^{\kappa-2}$. Thus we can think of

$$
D \equiv 2-\kappa=2-\frac{4 \pi}{3} \lambda
$$

as the fractal dimension of the unaffected portion of the "celestial" sphere at $\xi \rightarrow \infty$. Clearly, this is also the fractal dimension of the remaining surface of our bubble which has not been hit by other bubbles at future infinity.

\section{ANISOTROPIES IN THE DISTRIBUTION OF COLLISIONS}

To study collisions around points which are away from the origin, it is useful to perform a de Sitter transformation (Lorentz transformation in the embedding space) to a new frame $S^{\prime}$ where the point of interest is at the origin. This greatly simplifies the geometry of the relevant past light cones.

If the original point at large $\xi=\xi_{\text {obs }}$ was along the $Z$ direction, then we use the boost

$$
\begin{gathered}
V^{\prime}=\gamma(V-\beta Z), \quad Z^{\prime}=\gamma(Z-\beta V), \\
X^{\prime}=X, \quad Y^{\prime}=Y, \quad W^{\prime}=W,
\end{gathered}
$$


with $\beta=\tanh \xi_{\text {obs }}$ and $\gamma=\cosh \xi_{\text {obs }}$, in order to bring the point of interest down to $\xi_{\text {obs }}^{\prime}=0$. In doing so, the initial surface $t=t_{i}$ gets distorted and this is of course something we have to consider.

In the embedding coordinates, $t=t_{i}$ corresponds to the null plane

$$
W+V=e^{t_{i}} \equiv a_{i},
$$

which in boosted coordinates reads

$$
V^{\prime}+\beta Z^{\prime}=\gamma^{-1}\left(a_{i}-W^{\prime}\right) .
$$

We can now express this in terms of flat chart coordinates $\left(t^{\prime}, \vec{x}^{\prime}\right)$ by using the standard relations (5). This leads to

$$
\begin{aligned}
& \sinh t^{\prime}+\frac{1}{2} e^{t^{\prime}} r^{\prime 2}+\beta e^{t^{\prime}} r^{\prime} \cos \theta^{\prime} \\
& =\gamma^{-1}\left(a_{i}-\cosh t^{\prime}+\frac{1}{2} e^{t^{\prime}} r^{\prime 2}\right) .
\end{aligned}
$$

In terms of coordinates $\left(a^{\prime}, F^{\prime}\right)$ analogous to the $(a, F)$ pair which we introduced in the previous section [see Eq. (17)], the equation for the initial surface $a=a_{i}$ takes the more tractable form $a^{\prime}=a_{i}^{\prime}\left(F^{\prime}, \theta^{\prime} ; a_{i}\right)$, where

$$
a_{i}^{\prime}=2 \frac{a_{i}-\beta \gamma \cos \theta^{\prime}-(\gamma-1) F^{\prime}}{1+\gamma+2 \beta \gamma F^{\prime} \cos \theta^{\prime}+(\gamma-1) F^{\prime 2}} .
$$

It should be noted that $a_{i}^{\prime}$ can be negative for some values of $F^{\prime}$. This is not a problem. It just means that in the boosted frame, the initial surface invades the portion of de Sitter space not covered in the original chart.

It is interesting to calculate the probability that the FRW observer at $\xi^{\prime}=0$ (that is, $\xi=\xi_{\text {obs }}$ ) will be affected by a collision with another bubble before some specified time $\tau$. This is related to the space-time volume available for the nucleation of bubbles which lies in the past light cone of the observation point $\left(\xi^{\prime}=0, \tau\right)$, but which does not lie inside our reference bubble, or inside the past light cone of the nucleation event. This four volume increases with proper time, in the following way

$$
d V_{4}=\left[\int_{a_{i}^{\prime}}^{2 /\left(1-F^{\prime}\right)}\left(1+a^{\prime} F^{\prime}\right)^{2} d a^{\prime}\right] \frac{d F^{\prime}}{d \tau} d \tau d \Omega^{\prime},
$$

where $d \Omega^{\prime}=2 \pi d\left(\cos \theta^{\prime}\right)$. The probability per unit proper time and solid angle that our observer is hit by a bubble, assuming that he was not already hit by a bubble, is given by

$$
\frac{d P}{d \tau d \Omega^{\prime}}=\lambda \frac{d V_{4}}{d \tau d \Omega^{\prime}}
$$

From Eq. (13), $\xi^{\prime}=0$ corresponds to $F^{\prime}=-e^{-\tau}$, and performing the integration over $a^{\prime}$ we find

$$
\begin{aligned}
\frac{d V_{4}}{d \tau d \Omega^{\prime}}= & \frac{1}{3}\left[\left(\frac{\cosh \xi_{\mathrm{obs}} \sinh \tau+\cosh \tau-a_{i}}{\cosh \xi_{\mathrm{obs}} \cosh \tau+\sinh \tau-\sinh \xi_{\mathrm{obs}} \cos \theta^{\prime}}\right)^{3}\right. \\
& \left.-\tanh ^{3}\left(\frac{\tau}{2}\right)\right] .
\end{aligned}
$$

Note that the result depends both on the point of observation $\xi_{\text {obs }}$ and on the direction of observation $\theta^{\prime}$. Homogeneity and isotropy are lost, even in the limit when we push the initial surface all the way to $t_{i} \rightarrow-\infty$, i.e. $a_{i}=0$.

For any fixed nonzero value of $\tau$, the limit of (44) as $\xi_{\text {obs }} \rightarrow \infty$ is given by

$$
\frac{d V_{4}}{d \tau d \Omega^{\prime}}=\frac{1}{3}\left[\left(\frac{\sinh \tau}{\cosh \tau-\cos \theta^{\prime}}\right)^{3}-\tanh ^{3}\left(\frac{\tau}{2}\right)\right] .
$$

The existence of this limit is good news. The number of observers grows without bound with the distance to the center of the hyperboloid, and thus we expect that the typical observer lives at very large $\xi_{\text {obs }}$. He or she should therefore measure the distribution (45). Also, the limit is independent of $t_{i}$. This is also interesting, since it means that some specific details about the surface of initial conditions do not seem to matter. Nevertheless, the distribution is anisotropic, with the minimum number of hits per unit time in the direction of the center of our reference bubble. In this sense, memory of the initial surface persists. For large $\tau$, the distribution takes the simple dipole form

$$
\frac{d V_{4}}{d \tau d \Omega^{\prime}}=2\left(1+\cos \theta^{\prime}\right) e^{-\tau}+O\left(e^{-2 \tau}\right) .
$$

Dipole anisotropies and memory of initial conditions are not commodities one usually expects from inflation. Although we find this result to be rather shocking, the effect is real. Its origin is best understood by eliminating the complications due to the bubble geometry, as will be discussed in the next section. First, however, we would like to study a bit further the implications of (44).

To find the total rate $\lambda d V_{4} / d \tau$ at which bubbles will be encountered by an observer who has not previously been hit by a bubble, one can integrate (44) over solid angle. The result is given by

$$
\frac{d V_{4}}{d \tau}=\frac{4 \pi}{3}\left[\frac{\left(\cosh \xi_{\mathrm{obs}} \sinh \tau+\cosh \tau-a_{i}\right)^{3}\left(\cosh \xi_{\mathrm{obs}} \cosh \tau+\sinh \tau\right)}{\left(\cosh \xi_{\mathrm{obs}} \sinh \tau+\cosh \tau\right)^{4}}-\tanh ^{3}\left(\frac{\tau}{2}\right)\right]
$$


The dependence of this result on $\xi_{\text {obs }}$ shows us the inhomogeneity of the bubble collision rate. For the special case of $\tau=0$ the expression simplifies to

$$
\frac{d V_{4}}{d \tau}=\frac{4 \pi}{3}\left(1-a_{i}\right)^{3} \cosh \xi_{\mathrm{obs}}
$$

which is highly inhomogeneous, while for large $\tau$ the expression becomes

$$
\frac{d V_{4}}{d \tau}=8 \pi\left(1-\frac{a_{i}}{\cosh \xi_{\mathrm{obs}}+1}\right) e^{-\tau}+O\left(e^{-2 \tau}\right),
$$

which shows that the inhomogeneity disappears when $t_{i} \rightarrow$ $-\infty$ (i.e., $a_{i} \rightarrow 0$ ), and also when $\xi_{\text {obs }}$ is large.

We can continue by integrating (47) over $\tau$, from zero up to an arbitrary value, thereby determining the total 4 volume available for the nucleation of bubbles that could collide with an observer located at $\left(\xi_{\text {obs }}, \tau\right)$. One finds

$$
\begin{aligned}
V_{4}= & \frac{4 \pi}{3}\left\{\tanh ^{2}\left(\frac{\tau}{2}\right)-\ln \left[\epsilon \cosh ^{2}\left(\frac{\tau}{2}\right)\right]\right\} \\
& -4 \pi\left\{a_{i}(1-\epsilon)-\frac{1}{2} a_{i}^{2}\left(1-\epsilon^{2}\right)+\frac{1}{9} a_{i}^{3}\left(1-\epsilon^{3}\right)\right\},
\end{aligned}
$$

where

$$
\epsilon=\frac{1}{\cosh \xi_{\mathrm{obs}} \sinh \tau+\cosh \tau} .
$$

This quantity represents the 4 volume of the region that is in the past light cone of the point $\left(\xi_{\text {obs }}, \tau\right)$, but is not inside the reference bubble nor in the past light cone of its nucleation event. This volume can be calculated directly without making the coordinate transformation (37), and we have verified that the results agree.

If an observer at $\xi=\xi_{\text {obs }}$ has not seen a bubble at time $\tau_{1}$, then the probability that she will not be hit by a bubble by some later time $\tau_{2}$ is determined by (50), with

$$
P=\exp \left\{-\lambda\left[V_{4}\left(\xi_{\mathrm{obs}}, \tau_{2}\right)-V_{4}\left(\xi_{\mathrm{obs}}, \tau_{1}\right)\right]\right\} .
$$

It is particularly interesting to look at (50) for large $\xi_{\text {obs }}$, since a typical observer will be found at arbitrarily large $\xi_{\text {obs. }}$. In that limit one finds that

$$
\epsilon=\frac{2 e^{-\xi_{\mathrm{obs}}}}{\sinh \tau}-\frac{4 \cosh \tau}{\sinh ^{2} \tau} e^{-2 \xi_{\mathrm{obs}}}+O\left(e^{-3 \xi_{\mathrm{obs}}}\right),
$$

which is valid for any $\tau \neq 0$. Then

$$
\begin{aligned}
V_{4}= & \frac{4 \pi}{3}\left\{\xi_{\mathrm{obs}}+\tanh ^{2}\left(\frac{\tau}{2}\right)+\ln \left[\tanh \left(\frac{\tau}{2}\right)\right]\right. \\
& \left.+2 e^{-\xi_{\mathrm{obs}}} \operatorname{coth}(\tau)\right\} \\
& -4 \pi\left\{a_{i}-\frac{1}{2} a_{i}^{2}+\frac{1}{9} a_{i}^{3}-2 a_{i} \frac{e^{-\xi_{\mathrm{obs}}}}{\sinh \tau}\right\}+O\left(e^{-2 \xi_{\mathrm{obs}}}\right) .
\end{aligned}
$$

Then, if we are interested in the limit $\tau \rightarrow \infty$, we find

$$
\begin{aligned}
\lim _{\tau \rightarrow \infty} V_{4}= & \frac{4 \pi}{3}\left(\xi_{\mathrm{obs}}+1+2 e^{-\xi_{\mathrm{obs}}}\right)-4 \pi\left(a_{i}-\frac{1}{2} a_{i}^{2}+\frac{1}{9} a_{i}^{3}\right) \\
& +O\left(e^{-2 \xi_{\mathrm{obs}}}\right) .
\end{aligned}
$$

The probability of a point not being hit by a bubble is given by $P=\exp \left(-\lambda V_{4}\right)$, so the leading term $4 \pi \xi_{\text {obs }} / 3$ in the formula above reproduces Eq. (32), which was used to determine the fractal dimension.

\section{A MOVING OBSERVER IN DE SITTER SPACE}

Even for an observer in the false vacuum, the bubble nucleation rate and the angular distribution of bubbles depend on the observer's velocity relative to the "preferred" comoving congruence $\mathcal{C}$ which is determined by the surface of initial conditions. Here, we give a selfcontained account of this effect.

Consider a de Sitter space with $H=1$,

$$
d s^{2}=\eta^{-2}\left(d \eta^{2}-d \mathbf{x}^{2}\right),
$$

with $-\infty<\eta<0$. The conformal time $\eta$ is related to the usual time vatiable as

$$
\eta=-e^{-t}
$$

We shall assume that there are no bubbles at some initial moment $\eta=\eta_{i}$.

Consider an observer at $\mathbf{x}=0, \eta=\eta_{0}$, moving with a velocity

$$
v \equiv \tanh \phi
$$

relative to the comoving observers of (56). We want to know the probability for this observer to be hit by a bubble per unit proper time (by his clock).

An infinitesimal proper time interval $\delta \tau$ corresponds to a coordinate displacement

$$
\begin{gathered}
\delta \eta=\left|\eta_{0}\right| \delta \tau \cosh \phi, \\
\delta \mathbf{x}=\mathbf{v} \delta \eta .
\end{gathered}
$$

The probability to be hit by a bubble is determined by the space-time volume between the past light cones of the points $\left(\eta_{0}, 0\right)$ and $\left(\eta_{0}+\delta \eta, \delta \mathbf{x}\right)$. The first of these light cones is given by

$$
\left|\mathbf{x}_{1}(\eta)\right|=\eta_{0}-\eta
$$

and the second is given by

$$
\left|\mathbf{x}_{2}-\delta \mathbf{x}\right|=\delta \eta+\eta_{0}-\eta
$$

To linear order in $\delta \tau$,

$$
|\mathbf{x}-\delta \mathbf{x}| \approx r-\left|\eta_{0}\right| \delta \tau \sinh \phi \cos \theta=r-v \delta \eta \cos \theta,
$$

where $r=|\mathbf{x}|$ and $\theta$ is the angle between $\mathbf{x}$ and $\mathbf{v}$, so we can rewrite (62) as 


$$
r_{2}(\eta, \theta)=\delta \eta(1+v \cos \theta)+\eta_{0}-\eta .
$$

The space-time volume between the two light cones is given by the integral

$$
\begin{aligned}
\delta V_{4}= & 2 \pi \int_{\eta_{i}}^{\eta_{0}} d \eta \eta^{-4} \int_{0}^{\pi} d \theta \sin \theta r_{1}^{2}(\eta) \delta r(\theta) \\
= & 2 \pi \delta \eta \int_{\eta_{i}}^{\eta_{0}} d \eta \eta^{-4}\left(\eta_{0}-\eta\right)^{2} \\
& \times \int_{0}^{\pi} d \theta \sin \theta(1+v \cos \theta),
\end{aligned}
$$

where $\delta r(\theta)=r_{2}(\eta, \theta)-r_{1}(\eta)=\delta \eta(1+v \cos \theta)$. The integration over $\eta$ is easily done by a change of variable $\xi=-1 / \eta$

$$
\begin{aligned}
\int_{\eta_{i}}^{\eta_{0}} d \eta \eta^{-4}\left(\eta_{0}-\eta\right)^{2} & =\int d \xi\left(\eta_{0} \xi+1\right)^{2} \\
& =\frac{1}{3} \eta_{0}^{2}\left(\xi_{0}-\xi_{i}\right)^{3} .
\end{aligned}
$$

Substituting this into (65) and using Eq. (59) for $\delta \eta$, we have

$$
\delta V_{4}=\frac{2 \pi}{3} \delta \tau \cosh \phi f\left(t_{0}-t_{i}\right) \int_{0}^{\pi} d \theta \sin \theta(1+v \cos \theta),
$$

where

$$
f(t)=\left(1-e^{-t}\right)^{3}
$$

and $t$ is the usual time variable which is related to $\eta$ as in Eq. (57).

Note that $f(t) \rightarrow 1$ as $t \rightarrow \infty$. In what follows we assume the limit $t_{i} \rightarrow-\infty$ and set $f\left(t_{0}-t_{i}\right)=1$.

The $\theta$ integration in (67) is, of course, easily done; the result is

$$
\delta V_{4}=\frac{4 \pi}{3} \delta t
$$

where

$$
\delta t=\left|\eta_{0}\right|^{-1} \delta \eta=\delta \tau \cosh \phi
$$

is the interval of time $t$ corresponding to the proper time interval $\delta \tau$.

The result (69) is easy to understand. We have two past light cones, one has its origin at $\left(t_{0}, 0\right)$ and the other has its origin shifted in both time and space directions. Equation (69) tells us that the 4-volume difference $\delta V_{4}$ depends only on the time displacement $\delta t$. And this is as it should be. The volumes of the light cones do not change when we shift them in the "horizontal" (space) directions, so $\delta V_{4}$ remains unchanged, as long as one light cone remains entirely within the other.

All observers see the same bubble nucleation rate per unit time $t$, but this corresponds to different rates per unit proper time $\tau$,

$$
\frac{\delta V_{4}}{\delta \tau}=\frac{4 \pi}{3} \cosh \phi
$$

There is thus a preferred frame, where $\phi=0$, corresponding to the lowest nucleation rate.

The distribution of the arrival directions of the bubbles is also anisotropic. The angular distribution can be easily read from Eq. (67),

$$
\frac{d\left(\delta V_{4}\right)}{\delta \tau d \Omega}=\frac{1}{3} \cosh \phi(1+v \cos \theta) .
$$

The velocity of a geodesic observer relative to the comoving frame decays as

$$
v(t) \propto e^{-t}
$$

at large $t$, so the anisotropy rapidly disappears and the rate approaches that for a comoving observer. However, when we study bubble collisions and construct FRW coordinates inside a bubble, the set of comoving observers associated with these coordinates includes observers with arbitrarily large values of $v$. This is the origin of the anisotropy measured by typical observers in a bubble universe.

The frame dependence of the bubble arrival rate is perhaps not too surprising. To the moving observer the initial surface $t=t_{i} \rightarrow-\infty$ looks rather odd. It is a null surface which crossed the worldline of the observer a finite proper time $\tau$ in the past. This time has been calculated in [5]; it is given by

$$
\tau=\frac{1}{2} \ln \left(\frac{\gamma+1}{\gamma-1}\right) .
$$

For $(\gamma-1) \ll 1$, the initial surface is many Hubble times ago, and the difference from a comoving observer is small. However, for $\gamma \gg 1$, the cutoff surface is only a small fraction of the Hubble time away. No wonder it changes the rate and introduces an asymmetry.

The connection between (72) and the results of the previous section is not completely straightforward, and deserves some comment. The main difference is that observers inside the bubble will not have any bubbles nucleating in their immediate neighborhood (since we assume that bubbles can only nucleate in false vacuum).

Near the surface $\tau=0$, corresponding to the origin of time in the open chart, this difference becomes irrelevant. For $\tau=0$, Eq. (44) gives

$$
\frac{d V_{4}}{d \tau d \Omega^{\prime}}=\frac{1}{3 \gamma^{3}\left(1-\beta \cos \theta^{\prime}\right)^{3}} .
$$

At first sight, this looks rather different from (72). However, if we take into account the aberration of the angles in a moving frame,

$$
\cos \theta=\frac{\cos \theta^{\prime}-\beta}{1-\beta \cos \theta^{\prime}},
$$

we find 


$$
\frac{d V_{4}}{d \tau d \Omega}=\frac{1}{3} \gamma(1+\beta \cos \theta) .
$$

This agrees with (72) under the identifications $\gamma=\cosh \phi$ and $\beta=v$. From Eq. (8), the FRW observer at constant $\xi$ has a relativistic factor with respect to the preferred "rest frame" congruence $\mathcal{C}$ which is given by

$$
\gamma=\frac{d t}{d \tau}=\frac{\sinh \tau+\cosh \xi \cosh \tau}{\cosh \tau+\cosh \xi \sinh \tau} .
$$

For $\tau=0$, we have $\gamma=\cosh \xi$, which is unbounded for large $\xi$. Hence, right after nucleation, observers in this FRW congruence have arbitrarily large relativistic factors.

For any $\tau>0$, the limit of very large $\xi$ gives a finite $\gamma \approx$ $\operatorname{coth} \tau$. This means that a period of inflation inside the bubble slows down all observers to nonrelativistic speed (for $\tau \gg 1$, the velocity is of order $e^{-\tau}$ ). Nevertheless, to the observers at large $\xi$, the initial surface looks "slanted," sloping down in time in the direction away from the origin. This leads to the anisotropy in the distribution of bubbles. In embedding coordinates, the initial surface at $t=t_{i}$, is described as the intersection of the null surface $W+V=$ $e^{t_{i}}$ with the de Sitter hyperboloid (4) [the coordinate $V$ is "time," whereas the $W$ axis is normal to the hyperboloid at the nucleation point $(V=0, W=1)]$. Now, we can always bring an observer from very large $\xi$ to the origin of coordinates $\xi^{\prime}=0$ by means of a large boost, with $\beta=$ $\tanh \xi \approx 1$. If in the original "rest" frame the observer was at large $z$, the surface of initial conditions in the new reference frame takes the form $V^{\prime}+Z^{\prime} \approx 0$. This "initial" plane is (almost) tangent to the bubble cone, along its null generator in the negative $z^{\prime}$ direction. Since this leaves no room between the initial surface and the reference bubble, it is clear that the probability of being hit from that particular direction vanishes. From the point of view of the rest frame $\mathcal{C}$ the anisotropy is not surprising either. The observers at large $\xi$ are very close to the light cone, near the surface of the reference bubble. Other bubbles can only hit from the false vacuum outside, and because of that FRW observers are more likely to be hit by bubbles approaching from even larger $\xi$ than from any other direction.

\section{CONCLUSIONS}

Our primary goal in this project was to study the effect of bubble collisions on the structure of "bubble universes" in the inflating false vacuum. In particular, we wanted to know how likely it is for an observer living in one of the bubbles to be affected by such a collision.

In the absence of collisions, the bubble interior is described by an open FRW model. A constant-FRW-time slice of such an unperturbed bubble universe is a hyperboloid, a space of constant negative curvature. Each bubble collision carves an infinite wedgelike region out of the hyperboloid. We found that the part of the hyperboloid that remains unaffected by collisions has a fractal character. Its volume is infinite, but it constitutes a vanishing fraction of the total volume of the hyperboloid.

If we pick a random observer in the unaffected region, then we find that the typical distance $d$ from this observer to the nearest bubble collision is given by

$$
d \sim R \ln (1 / \lambda) .
$$

Here $R$ is the curvature radius of the hyperboloid and $\lambda \ll$ 1 is the bubble nucleation rate (per Hubble volume per Hubble time in the false vacuum). The origin of the logarithmic dependence on $\lambda$ is that the volume grows exponentially with distance on a hyperboloid. Current observational bounds on the spatial curvature imply $R \geqslant$ $10 H_{0}^{-1}$, where $H_{0}^{-1}$ is the present Hubble radius, and Eq. (76) yields $d>10 H_{0}^{-1}$.

Assuming that we live in a bubble universe, we have also estimated the probability for a collision to occur within our observable range. This is given by

$$
P_{\text {coll }} \sim 4 \pi \lambda / H_{0} R \lesssim \lambda .
$$

The bubble nucleation rate $\lambda$ is usually exponentially suppressed, and thus the chance for us to observe a bubble collision is rather remote.

In the process of this investigation, we have uncovered a remarkable fact, that the probability for an observer to be hit by a bubble has a strong dependence on the arrival direction of the bubble. The origin of this effect can be traced to a simpler setting, which does not involve bubble collisions. Consider a geodesic observer who lives in false vacuum. We want to know the probability for this observer to be hit by a bubble, per unit time by his clock. We found that this probability depends on the observer's velocity $\mathbf{v}$ relative to a certain preferred "comoving" frame, which is set by the initial conditions at the beginning of inflation. The bubble nucleation rate is minimal for comoving observers with $\mathbf{v}=0$, and the most probable arrival direction for a bubble is that opposite to $\mathbf{v}$.

For a single observer, these effects will be present only for a brief period of time. If the initial state of false vacuum is specified on some spacelike hypersurface, the comoving frame is defined by the congruence $\mathcal{C}$ of geodesics orthogonal to that surface. The velocity of a geodesic observer relative to $\mathcal{C}$ redshifts exponentially with time, so the bubble nucleation rate rapidly becomes isotropic and approaches its comoving value. This is in accord with the widespread belief that the initial conditions at the onset of inflation have no lasting effect.

We found, however, that this folk wisdom does not apply when the situation is described in terms of a FRW open universe inside the bubble. The geodesic congruence corresponding to such a universe includes geodesics with velocities arbitrarily close to the speed of light relative to $\mathcal{C}$. Fast moving observers in this congruence would initially detect an extremely high rate of bubble hits. The short 
period of inflation inside the bubble slows down the congruence to nonrelativistic speeds, and the rate approaches a constant on the hyperboloid (at large distances from the origin). Nevertheless, the angular asymmetry in the arrival directions of the bubbles remains. The reason is that, to an observer far from the origin, the initial surface looks very anisotropic, sloping down in time in the radial direction further away from the origin. In the unlikely event that we detect a signature of a bubble collision in some direction in the sky, we will be able to say that we are probably moving in that general direction relative to the preferred congruence $\mathcal{C}$.

Now that we know that the initial conditions at the beginning of inflation have a lasting effect on the distribution of bubble hits, one cannot help wondering what other effects they may have. We hope to return to this issue in the future.

\section{ACKNOWLEDGMENTS}

The work of J. G. was supported in part by CICYT and DURSI. The work of A. V. was supported in part by Grant No. PHY-0353314 from The National Science Foundation and by Grant No. RFP1-06-028 from The Foundational Questions Institute. The work of A. H. G. was supported in part by the U.S. Department of Energy (D.O.E.) under cooperative research agreement No. DF-FC0294ER40818, and also by the Kavli Foundation.

\section{APPENDIX A: COLLISIONS AT $\xi_{\text {obs }} \gg 1$}

In this appendix, we repeat the calculation of the distribution of collisions which we presented in Sec. II A, but now instead of looking around the origin we consider the vicinity of a point $\xi_{\text {obs }} \gg 1$.

It is useful to go to the boosted frame where the point of observation is at the origin, as we did in Sec. II. Furthermore, we may take the limit $\gamma=\cosh \xi_{\text {obs }} \rightarrow \infty$, $\beta=\tanh \xi_{\mathrm{obs}} \rightarrow 1$ in the expression for the initial surface $(41)$,

$$
a_{i}^{\prime}=-2 \frac{\cos \theta^{\prime}+F^{\prime}}{1+2 F^{\prime} \cos \theta^{\prime}+F^{\prime 2}} .
$$

We may take Eq. (42) as the starting point. Performing the integral over $a^{\prime}$ we have

$$
\begin{aligned}
d V_{4}= & \frac{1}{3 F^{\prime}}\left[\left(\frac{1+F^{\prime}}{1-F^{\prime}}\right)^{3}\right. \\
& \left.-\left(\frac{1-F^{\prime 2}}{1+2 F^{\prime} \cos \theta^{\prime}+F^{\prime 2}}\right)^{3}\right] d F^{\prime} d \Omega^{\prime} .
\end{aligned}
$$

Integrating over solid angle, we have

$$
d V_{4}=\frac{8 \pi\left(3+2 F^{\prime}+3 F^{\prime 2}\right)}{3\left(1-F^{\prime}\right)^{3}\left(1+F^{\prime}\right)} d F^{\prime} .
$$

Finally, integrating over $F^{\prime}$ we obtain

$$
\begin{aligned}
& V_{4}\left(\xi^{\prime}, \tau, t_{i} \rightarrow-\infty, \xi_{\mathrm{obs}} \gg 1\right) \\
& \quad=\frac{4 \pi}{3}\left[\frac{4 F^{\prime}}{\left(1-F^{\prime}\right)^{2}}-\ln \frac{1-F^{\prime}}{1+F^{\prime}}\right]_{-e^{-\tau}}^{F^{\prime}\left(\xi^{\prime}, \tau\right)}
\end{aligned}
$$

After some algebra, this greatly simplifies to

$$
V_{4}\left(\xi^{\prime}, \tau, \xi_{\text {obs }} \gg 1\right)=\frac{4 \pi}{3}\left[2 e^{\xi^{\prime}} \sinh \xi^{\prime} \tanh ^{2}(\tau / 2)+\xi^{\prime}\right] .
$$

This can be compared with the distribution around the privileged point $\xi_{\text {obs }}=0$ given in (21). The expressions are rather similar. In particular the asymptotic expressions $(\tau \gg 1)$

$$
\begin{aligned}
& V_{4} \approx 4 \pi \xi^{\prime}+O\left(\xi^{2}\right) \quad\left(\xi^{\prime} \ll 1\right), \\
& V_{4} \approx \frac{4 \pi}{3} e^{2 \xi^{\prime}}+O\left(\xi^{\prime}\right) \quad\left(\xi^{\prime} \gg 1\right) .
\end{aligned}
$$

are the same.

Suppose we match the inflating phase to a standard cosmological phase with scale factor given by $a(\tau)$. As the universe decelerates, the horizon becomes larger and more bubbles come into sight. We can then ask what is the rate at which bubbles become visible per unit time to the observer at $\xi^{\prime}=0$. This is proportional to the nucleation rate $\lambda$, times the rate at which the relevant 4 volume enters the backward light cone from the point of observation:

$$
\frac{d N(\tau)}{d \tau}=\left.\lambda \frac{\partial V_{4}}{\partial \xi^{\prime}}\right|_{\tau=\tau_{e}, \xi^{\prime}=\xi^{\prime}(\tau)} \frac{d \xi^{\prime}(\tau)}{d \tau} .
$$

Here $\tau_{e}$ is the time at the end of inflation, and the last factor gives the rate at which the observable distance changes as we look back to the surface where inflation ends:

$$
\xi(\tau)=\int_{\tau_{e}}^{\tau} \frac{d \tau}{a(\tau)} .
$$

For instance, if inside the bubble the vacuum energy is zero, and we go immediately into the curvature dominated regime $a(\tau)=\tau$, then the rate at which we would see new bubbles entering our horizon per unit proper time is given by

$$
\frac{d N(\tau)}{d \tau}=\frac{4 \pi \lambda}{3}\left[\frac{\tau}{2}+\frac{1}{\tau}\right]
$$

Since this grows without bound, we would be guaranteed to see some bubbles sooner or later if we lived in this Minkowski vacuum.

\section{APPENDIX B: SPECIAL CONFIGURATIONS}

Here, we explore the possibility of setting up initial conditions which do not break the residual $O(3,1)$ symmetry of the bubble. This is a formal exercise whose practical utility is unclear. Bubbles nucleate at random points, and it is not possible to set up initial conditions which preserve $O(3,1)$ symmetry for all of the bubbles in 
the ensemble. Nevertheless, the attempt may be illustrative.

The simplest thing we can do in order to have $O(3,1)$ invariant initial conditions, is to require that there are no bubbles inside the backward light cone from the antipodal point A of the nucleation event $\mathrm{N}$ (see Fig. 2). We are also assuming that $\mathrm{N}$ is in false vacuum, so the surface of initial conditions is the disjoint union of the backward light cones from $\mathrm{A}$ and $\mathrm{N}$. The relevant four-volume of our interest is represented by the shaded area in Fig. 2.

It is convenient to use the conformal closed chart, in which the metric reads

$$
d s^{2}=\frac{1}{\cos ^{2} \eta}\left(-d \eta^{2}+d \chi^{2}+\sin ^{2} \chi d \Omega^{2}\right) .
$$

The 4 volume is given by

$$
\begin{aligned}
\tilde{V}_{4}(\xi, \tau) & =4 \pi \int \frac{\sin ^{2} \chi}{\cos ^{4} \eta} d \chi d \eta \\
& =8 \pi \int_{u_{\min }}^{u_{\max }} d u \int_{0}^{\pi / 2} d v \frac{\sin ^{2}(v-u)}{\cos ^{4}(v+u)},
\end{aligned}
$$

where we have introduced the change of coordinates $\eta=$ $v+u$ and $\chi=v-u$. Past light cones are labeled by $u=$ const values. Performing the integrals, we have

$$
\tilde{V}_{4}(\xi, \tau)=\frac{8 \pi}{3}\left[\frac{2 \cos 2 u}{\sin ^{2} 2 u}-\operatorname{lntan}^{2} u\right]_{u_{\min }}^{u_{\max }} .
$$

Finally, we must relate the values of $u$ to the values of $\xi$ on the $\tau=$ const hyperboloid. Alternatively, we may relate them to the by-now familiar variable $F$.

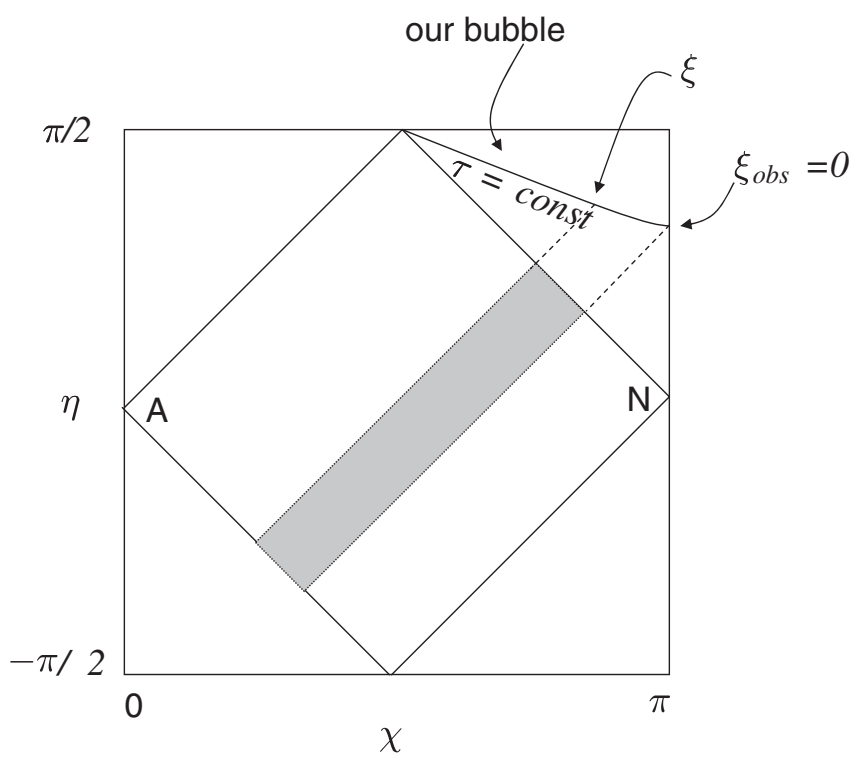

FIG. 2. Conformal diagram of a bubble in a de Sitter space. The relevant 4 volume to the past of a sphere of radius $\xi$ around the origin is shaded in gray.
On the bubble cone we have $\chi=\pi-\eta$, and hence the physical radius of two-spheres is given by

$$
R=\frac{\sin \chi}{\cos \eta}=\tan \eta=-\cot u .
$$

On the other hand, in terms of $a$ and $F$ coordinates we have

$$
R=(1+a F)=\frac{1+F}{1-F},
$$

where we have used that on the bubble cone $a=2 /(1-$ $F$ ) [see Eq. (15)]. Equating both expressions for $R$ we have

$$
\tan u=\frac{F-1}{F+1} .
$$

Substituting in (B3) we obtain

$$
\tilde{V}_{4}=\frac{8 \pi}{3}\left[\frac{4 F\left(F^{2}+1\right)}{\left(F^{2}-1\right)^{2}}-2 \ln \frac{1-F}{1+F}\right]_{-e^{-\tau}}^{F(\xi \tau)} .
$$

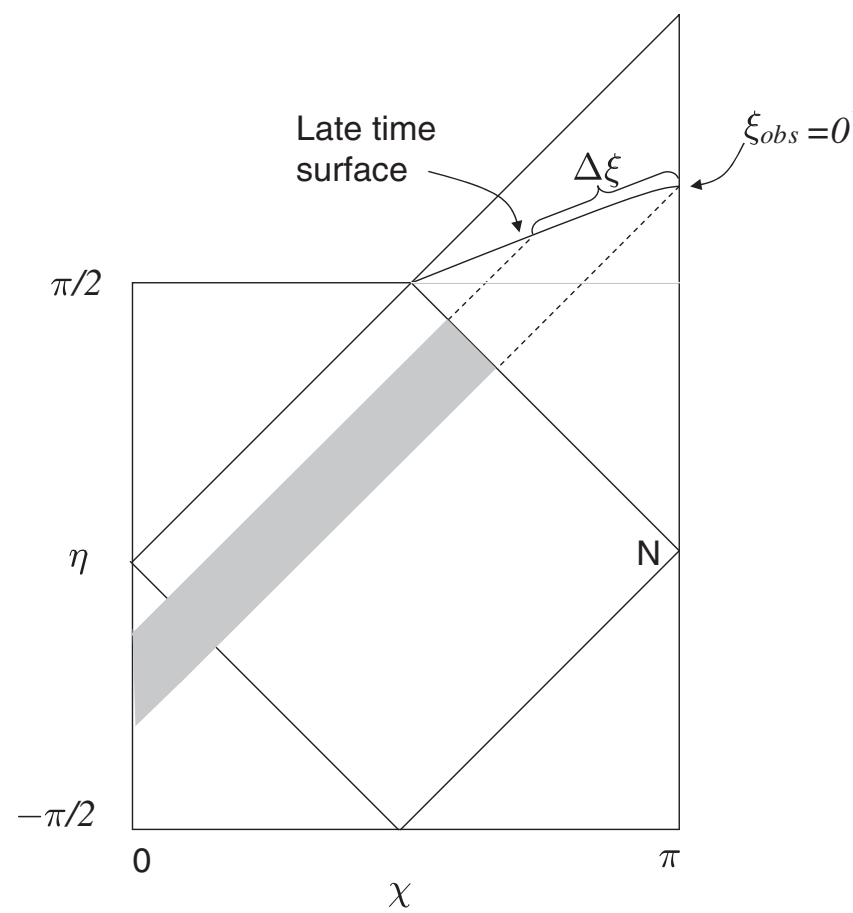

FIG. 3. Same as in Fig. 2, but now the true vacuum inside the bubble is of lower energy. For simplicity, we take it to be Minkowski, although this is not essential. In this case, the shaded area corresponding to the 4 volume available for the nucleation of bubbles which will hit a distance $\Delta \xi$ away from the point of observation, is finite. All we need is that the backward light cone from the point of observation reaches the point $\chi=0$ at some time in the past. This will happen if the cosmological horizon at the time of observation is many times larger than the Hubble size during inflation. Formally, we do not need a cutoff initial surface, and seemingly we obtain a finite answer which respects $O(3,1)$ invariance. However, we know that physically some initial conditions are needed. These look different to different observers in the FRW congruence, which leads to anisotropies in the distribution of bubbles. 
Note that the leading dependence for large $\xi$ is the same as in $V_{4}$, given in (20). However, both expressions differ in the subleading terms, and so the limit of large $\xi_{\text {obs }}$ studied in the previous section does not exactly agree with the result which we have just obtained by setting up Lorentz invariant initial conditions.

Formally, we have obtained a finite result which does not break the homogeneity and isotropy of the reference bubble, by assuming that the point of observation is still in false vacuum. However, it is clear that the 4 volume $\tilde{V}_{4}$ diverges when we set $\tau=0$ in the lower limit of integration. What this means is that the bubble has probability 1 of being hit by other bubbles immediately after its formation. In other words, there is no possibility of eternal inflation with these initial conditions: we have included too much of the contracting part of de Sitter space.

Finally, we could have adopted yet another approach which formally does not require any "cutoff" initial surface. Indeed, we could ask the following question. Given that a particular point has not been hit by a bubble at some late time in the true vacuum (e.g. by the time of last scattering), what is the expected distance to the nearest bubble? If the true vacuum is of sufficiently low energy, the relevant 4 volume is finite, without the need of any cutoff. This is illustrated in Fig. 3. For clarity, we draw the case where the "true" vacuum inside the bubble is Minkowski. In practice, it is enough that the true vacuum be of sufficiently low energy density, so that the observer's cosmological horizon is much bigger than the Hubble size during inflation. In this case, the shaded area corresponding to the 4 volume available for the nucleation of bubbles which will hit a distance $\Delta \xi$ away from the point of observation, is finite. Formally, we avoid the need to specify the initial surface.

Again, this formal setup is unphysical. Some initial conditions must be specified, and these will look different from the point of view of different observers. This will lead to the anisotropies which we have discussed in the present paper.
[1] R. Bousso and J. Polchinski, J. High Energy Phys. 06 (2000) 006.

[2] L. Susskind, in Universe or Multiverse, edited by B.J. Carr (Cambridge University Press, Cambridge, U.K., 2006).

[3] S. Coleman and F. De Luccia, Phys. Rev. D 21, 3305 (1980).

[4] This version of eternal inflation has been discussed in J. R. Gott, Nature (London) 295, 304 (1982); A. H. Guth and E. Weinberg, Nucl. Phys. B212, 321 (1983); P. J. Steinhardt, in The Very Early Universe, edited by G. W. Gibbons,
S. W. Hawking, and S. T. C. Siklos (Cambridge University Press, Cambridge, U.K., 1983); J. R. Gott and T. S. Statler, Phys. Lett. B 136, 157 (1984); M. Bucher, A. S. Goldhaber, and N. Turok, Phys. Rev. D 52, 3314 (1995); K. Yamamoto, M. Sasaki, and T. Tanaka, Astrophys. J. 455, 412 (1995).

[5] A. Borde, A. H. Guth, and A. Vilenkin, Phys. Rev. Lett. 90, 151301 (2003).

[6] D. N. Spergel et al., Astrophys. J. Suppl. Ser. 170, 377 (2007). 